\title{
DESARROLLO Y APLICACIÓN DE UN ÍNDICE MULTIFACTORIAL DE CONECTIVIDAD DE SEDIMENTOS A ESCALA DE CUENCA
}

\author{
J.M. QUIÑONERO-RUBIO ${ }^{1,2 *}$, \\ C. BOIX-FAYOS ${ }^{2}$, J. DE VENTE ${ }^{2}$
}

\author{
${ }^{1}$ Departamento de Geografía, Universidad de Murcia, Campus de La Merced, 30001 Murcia. \\ ${ }^{2}$ Departamento de Conservación de Suelos y Agua y Manejo de Residuos Orgánicos, Grupo de Erosión \\ y Conservación de Suelos, CEBAS-CSIC, Campus de Espinardo, 30100 Espinardo (Murcia).
}

RESUMEN. Este trabajo presenta una nueva metodología para la estimación de la conectividad de sedimentos a escala de cuenca. El índice propuesto CCI (Índice de Conectividad de Cuenca), se basa en la combinación de factores en un entorno SIG que condicionan la conectividad de sedimentos en diferentes elementos (laderas, subcuencas, cauces) que componen el sistema fluvial. Los factores evaluados en el modelo son la capacidad de transporte en laderas (TC), la eficiencia como trampa de diques de retención de sedimentos (TE), la presencia de barreras geomorfológicas (GF), las condiciones del flujo (FC) y la capacidad de transporte de los cauces (SP). Aplicamos este índice en la cuenca del Alto Taibilla $\left(314 \mathrm{~km}^{2}\right)(\mathrm{SE}$ de España) para distintos escenarios de uso del suelo (1956 y 2006) y de manejo (diques de retención de sedimentos). Esta cuenca ha sufrido importantes cambios de cobertura en los últimos 50 años. El intenso proceso de abandono agrícola y la implantación de reforestaciones y diques de retención de sedimentos han causado un importante impacto en la dinámica de exportación de sedimentos de la cuenca. El CCI permite identificar qué elementos del paisaje tienen un mayor impacto en la (des)conectividad de sedimentos a escala de cuenca. Los resultados muestran una reducción importante de la conectividad del 76\% entre 1956 y 2006. Sin embargo, se observa que los diques de retención de sedimentos sólo contribuyen a esta reducción en un 3\%. Los cambios de uso del suelo provocan un impacto elevado en la reducción de la conectividad de sedimentos, salvo en sectores muy localizados con fuertes pendientes, o bien con el desarrollo de la agricultura junto a los principales cauces de la red de drenaje. El CCI resulta un método contrastado de fácil aplicación que puede ser utilizado para realizar análisis espacio-temporales de la conectividad de sedimentos en áreas con alteraciones naturales y antrópicas.

Development and application of a multi-factorial sediment connectivity index at the catchment scale

ABSTRACT. This paper presents a new methodology to assess sediment connectivity at the catchment scale. The proposed index CCI (Catchment 
Connectivity Index) is based on a combination of factors in a GIS environment that determine the connectivity of sediment in different elements (slopes, basins, channels) of the river system. The factors evaluated are the transport capacity on hillslopes (TC), check-dams' trap efficiency (TE), the presence of geomorphological barriers (GF), flow conditions (FC) and the sediment transport capacity in channels (SP). This index was applied to estimate connectivity in the Upper Taibilla catchment (314 $\mathrm{km}^{2}$ ) (SE Spain) for different land use (1956 and 2006) and management (check-dams) scenarios. This catchment has suffered major land use and land cover changes over the last 50 years. The intense agricultural abandonment process and the implementation of soil erosion control measures (reforestation and check-dams) have strongly affected sediment dynamics of the basin. Calculation of the CCI allows identifying which landscape elements have most impact on the sediment (dis)connectivity at catchment scale. The results show a significant reduction in the connectivity of 76\% between 1956 and 2006. However, it is observed that the check-dams contribute only $3 \%$ to this reduction. Land use changes had a much higher impact on reducing sediment connectivity, except in some areas with steep slopes, or with the development of agriculture along the main channels of the drainage network. Altogether, CCI showed to be a relatively easy and effective method that can be used for spatio-temporal sediment connectivity analyses in areas with natural and human disturbances.

Palabras clave: transporte de sedimentos, factores geomórficos, índice de conectividad, dique, cambios de uso del suelo.

Key words: sediment transport, geomorphological factors, connectivity index, check-dams, land use changes.

Enviado el 23 de noviembre de 2012

Aceptado el 29 de enero de 2013

*Correspondencia: Departamento de Geografía, Universidad de Murcia, Campus de La Merced, 30001 Murcia.E-mail: juanmaqr@um.es

\section{Introducción}

La conectividad de sedimentos en un sistema fluvial depende de la variabilidad espacial, organización espacial y conectividad interna de los elementos que componen el paisaje, así como de su capacidad de adaptación y tipo de respuesta ante cualquier factor de cambio (Hooke, 2003; Kasai et al., 2005; Borselli et al., 2007). De este modo, es posible demostrar la complejidad general y la heterogeneidad del funcionamiento espaciotemporal de un sistema fluvial determinado, desde las relaciones entre sus componentes, la localización de éstos y su extensión (Brierley et al., 2006). En la última década se ha mostrado desde la comunidad científica un especial interés en el estudio de la conectividad sedimentológica, evaluando el grado de la misma, desde las laderas y fuentes de sedimentos, transporte y almacenamiento a lo largo de los cauces hasta la capacidad de evacuación en los puntos de desembocadura (Hooke, 2006), considerando una jerarqui- 
zación de escalas (Cammeraat, 2002). Además, la identificación de barreras fluviales en los cauces, tales como diques de retención de sedimentos, áreas de deposición de sedimentos entre laderas y cauces, así como los cambios en el área de drenaje son en parte responsables de la evolución de la conexión ladera-cauce (coupling), la evolución de la morfología de cauces (incisión, erosión lateral, estrechamiento, agradación, degradación), y de los cambios en el balance de exportación de sedimentos a escala de cuenca.

Desde la década de 1990 el número de estudios sobre conectividad a escala de cuenca ha experimentado un incremento importante (Michaelides y Wainwright, 2002; LópezVicente et al., 2011). Se han extendido ampliamente los trabajos orientados a evaluar y cuantificar la producción y almacenamiento de sedimentos y escorrentía en los distintos compartimentos de una cuenca, pudiéndose clasificar en tres líneas de investigación: i) aquellos estudios con especial interés en la modelización de la erosión y/o escorrentía a escala de cuenca, que establecen una evolución de los usos del suelo a partir de la definición de distintos escenarios. Frecuentemente, estos trabajos analizan a su vez la eficiencia de estructuras artificiales como diques o terrazas de cultivo, así como el impacto que éstas provocan (Kasai et al., 2005; Lesschen et al., 2007; Boix-Fayos et al., 2008; Keesstra et al., 2009; Meerkerk et al., 2009; López-Vicente et al., 2011; Sougnez et al., 2011); ii) otros estudios están orientados a analizar los procesos de conexión (coupling) entre laderas y cauces, a partir de modelización hidrológica, o bien en la identificación y análisis de geoformas entre ambos elementos (Fryirs y Brierley, 1999; Harvey, 2002; Michaelides y Wainwright, 2002); iii) por último, aquellos estudios que evalúan las respuestas y cambios morfológicos de los cauces a partir de las fuentes y flujos de sedimentos, permanencia y distribución de éstos dentro de los cauces, y el impacto que los diques de retención de sedimentos provocan tanto aguas arriba como aguas abajo de su ubicación (Hooke, 2003; Fryirs y Brierley, 2001; Boix-Fayos et al., 2007; Sandercock y Hooke, 2011).

Sin embargo, pocos trabajos de investigación cuantifican la conectividad sedimentológica e hidrológica de una cuenca siguiendo un enfoque integral. En los últimos años destacan aquellos trabajos de Borselli et al. (2008), Lopez-Vicente et al. (2011) y Vigiak et al. (2012), que han aplicado índices de conectividad, relativamente complejos basados en la modelización y cuantificación de la conectividad hidrológica y de sedimentos de una cuenca con el fin de monitorizar los cambios de conectividad en áreas que sufren intensos cambios geomorfológicos o alteraciones antrópicas. Mientras, otros autores focalizan sus trabajos hacia la comprensión de la conectividad hidrológica con el fin de determinar la influencia espacio-temporal de la escorrentía sobre procesos geomórficos (Kirkby et al., 2002; Hooke et al., 2003; Bracken y Croke, 2007; Reaney et al., 2007; Reaney, 2008; Cantón et al., 2011). La frecuencia de avenidas y los factores que controlan la respuesta hidrológica de una cuenca reflejan las características del área vertiente y las fuentes potenciales de sedimentos (García-Ruiz et al., 2010). Complementariamente, un interesante enfoque geomorfológico está siendo desarrollado en la última década, teniendo en cuenta las condiciones que presentan los ríos y la conectividad a escala de cuenca, a partir de la identificación de elementos geomórficos del paisaje (Brierley et al., 2006; Fryirs et al., 2007a, b). La integración de elementos de los distintos enfoques hacia un análisis holístico de la conectividad podría significar un avance en la caracterización 
y el entendimiento del movimiento de sedimentos a escala de cuenca, ya que el análisis individualizado de determinados procesos o variables no refleja fielmente el grado de conectividad de una cuenca (García-Ruiz et al., 2010). Para ello el desarrollo de métodos de modelización espacial que se basen en procedimientos de trabajo de campo puede ayudar a calibrar y validar los conceptos en que se basa la conectividad (Bracken y Croke, 2007). Este trabajo tiene como objetivo general evaluar la conectividad de los sedimentos a escala de cuenca en un paisaje mediterráneo afectado por importantes cambios de uso de suelo. El estudio se centra en analizar cómo los componentes hidro-geomorfológicos de origen natural y las medidas de gestión introducidas por parte del hombre han causado cambios en la conectividad sedimentológica de la cuenca del Alto Taibilla entre 1956 y 2006. Los objetivos específicos son: i) desarrollar una metodología que permita integrar diferentes factores hidro-geomórficos para determinar la conectividad sedimentológica de la cuenca del Alto Taibilla; ii) realizar una valoración comparativa de la conectividad sedimentológica en la cuenca de estudio con varios escenarios de uso y manejo del suelo (1956 y 2006 con y sin diques de retención de sedimentos).

\section{2. Área de estudio}

La cuenca del río Taibilla está localizada en el límite entre la Región de Murcia y Albacete (sureste de España). Con un área total de $600 \mathrm{~km}^{2}$ representa uno de los afluentes más importantes de la cuenca del río Segura. Es una cuenca de montaña mediterránea perteneciente a la zona Subbética de las cordilleras Béticas, con una altitud entre los $800 \mathrm{~m}$ y los $2080 \mathrm{~m}$. El embalse del Taibilla se encuentra, aproximadamente, en el sector central de la cuenca, y ha sido identificado como desembocadura de la que se ha denominado en este trabajo cuenca del Alto Taibilla. Esta subcuenca de cabecera tiene una superficie de $314 \mathrm{~km}^{2}$ (Fig. 1) en la que la litología dominante se compone de calizas en las zonas montañosas y margas en valles, además de dolomías, margocalizas, areniscas, todas ellas del Cretácico, Oligoceno y Mioceno (IGME, 1980). El clima de la zona se considera mediterráneo subhúmedo en transición al semiárido, con una precipitación media anual de $500 \mathrm{~mm}$ y una temperatura media de $13^{\circ} \mathrm{C}$ (Boix-Fayos et al., 2007).

Desde la década de 1950 se ha experimentado en la zona un éxodo rural importante provocando cambios en la estructura del paisaje. El principal cambio identificado es el progresivo abandono de las zonas agrícolas y un incremento de las zonas boscosas. El incremento de la superficie de bosque se debe, no sólo a la revegetación de las zonas agrícolas por procesos naturales, sino también a proyectos de restauración hidrológico-forestal que incluían construcción de diques de retención de sedimentos en cauces (120 en la cuenca del Alto Taibilla, de los cuales se consideran 94 para este estudio) y reforestación de las cabeceras de las cuencas desde la década de 1970 (Boix-Fayos et al., 2007).

La restauración hidrológico-forestal, junto a la evolución experimentada por los usos del suelo han provocado importantes cambios en la dinámica de los flujos de agua y sedimentos dentro de esta cuenca. En general se ha identificado una disminución del caudal y del aporte de sedimentos desde las laderas induciendo ajustes fluviales a través de incisión, erosión del lecho y erosión lateral (Boix-Fayos et al., 2007; 2008). 


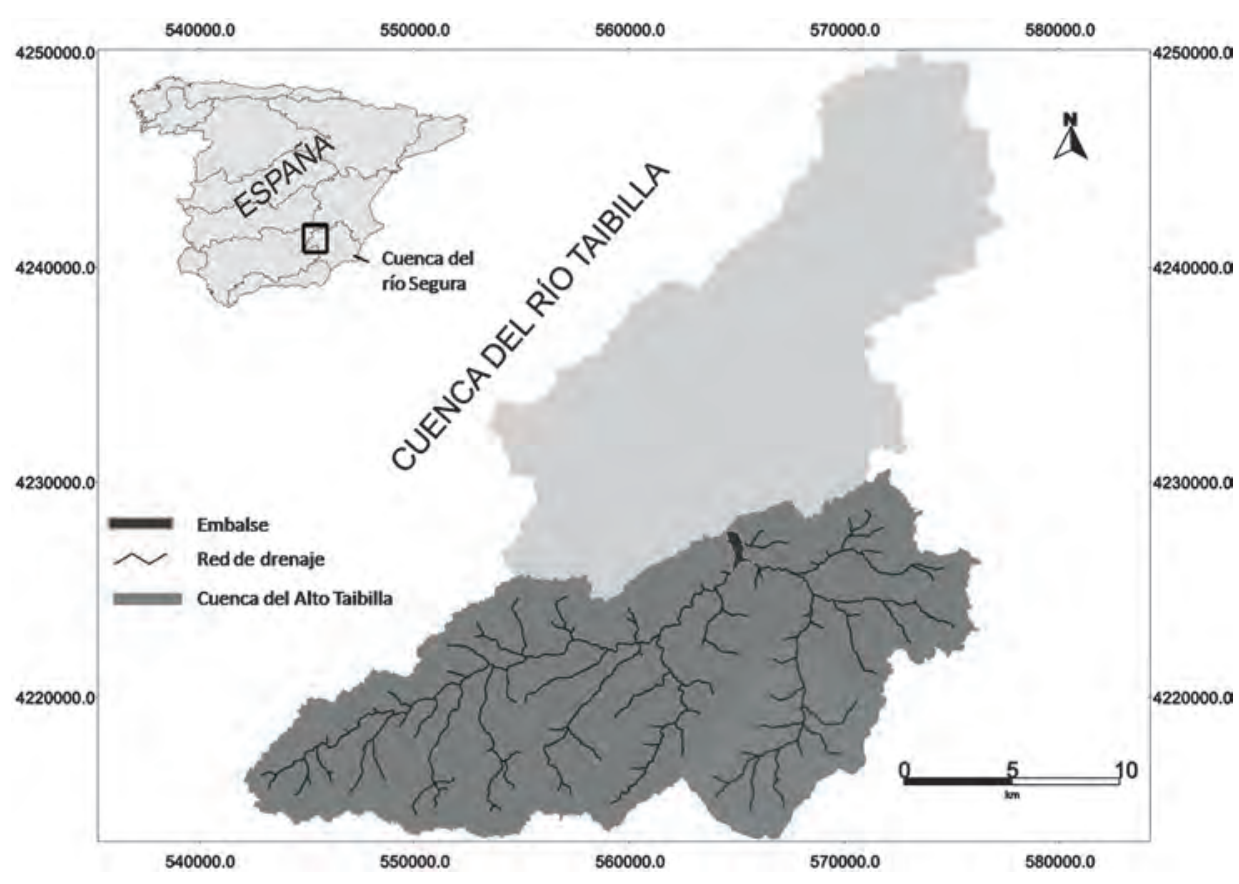

Figura 1. Localización de la cuenca del Alto Taibilla y su red de drenaje principal.

\section{Materiales y métodos}

En este trabajo se desarrolla una metodología de modelización espacio-temporal basada en factores hidro-geomorfológicos (Tabla 1) con el fin de determinar la conectividad de sedimentos a escala de cuenca. Proponemos un índice de conectividad basado en la valoración semi-cuantitativa de factores, a través de observaciones basadas en teledetección (análisis de fotografía aérea), modelización (modelo WaTEM-SEDEM [Van Oost et al., 2000; Van Rompaey et al., 2001; Verstraeten et al., 2002; de Vente et al., 2008]) y análisis con Sistemas de Información Geográfica y observaciones en el campo. Se establece así la identificación de los principales elementos del paisaje que puedan inducir cambios en la conectividad sedimentológica del Alto Taibilla. De este modo, se define el Índice de Conectividad de Cuenca (CCI) como:

$$
C C I=\left(\frac{T C_{w v}}{T C_{\max }}\right) \cdot\left(\frac{100-T E_{a v}}{100}\right) \cdot\left(\frac{G F_{u v}}{G F_{\max }}\right) \cdot\left(\frac{S P_{a v}}{S P_{a v_{-} \max }}\right) \cdot\left(\frac{F C_{a v}}{F C_{a v_{-} \max }}\right)
$$

donde TC (Transport Capacity) es la capacidad de transporte de sedimentos en laderas, TE (Trap Efficiency) es la capacidad de retención de sedimentos de los diques, GF (Geomorphological Factor) es el factor geomorfológico, SP (Stream Power) 


\begin{tabular}{|c|c|c|c|c|c|c|}
\hline & है & $\begin{array}{l}\text { है } \\
\text { है }\end{array}$ & 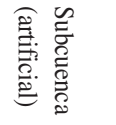 & 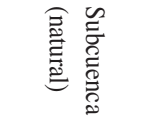 & 芯 & 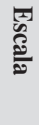 \\
\hline (1) & 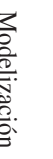 & 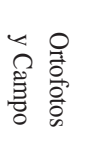 & 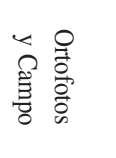 & 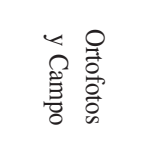 & 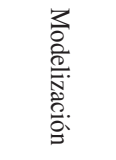 & $\begin{array}{l}\frac{a}{0} \\
\text { है }\end{array}$ \\
\hline 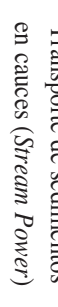 & 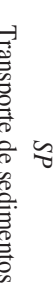 & 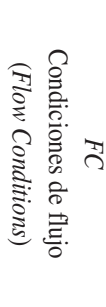 & 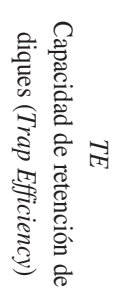 & 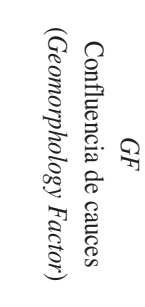 & 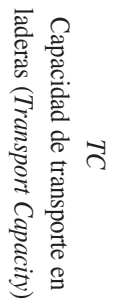 & 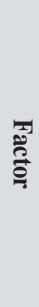 \\
\hline 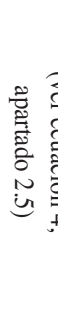 & 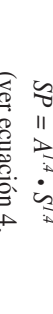 & 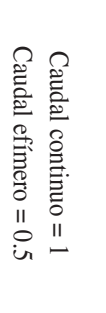 & 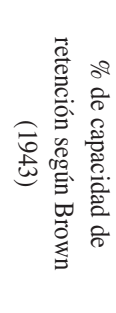 & 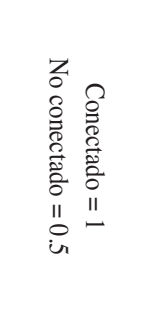 & 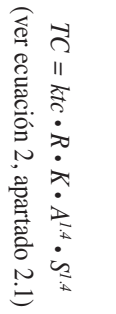 & 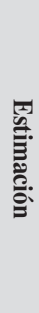 \\
\hline 2 & है. & 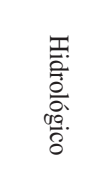 & 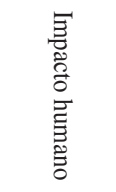 & 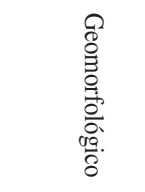 & 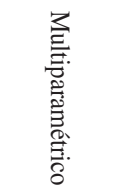 & 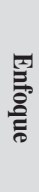 \\
\hline 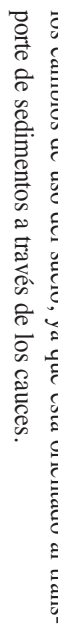 & 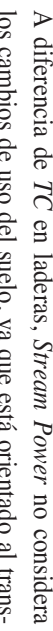 & 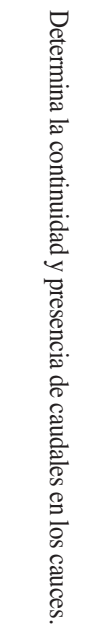 & 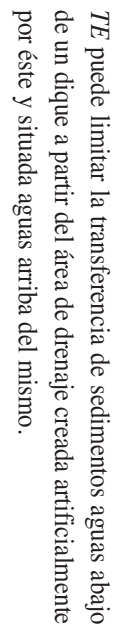 & 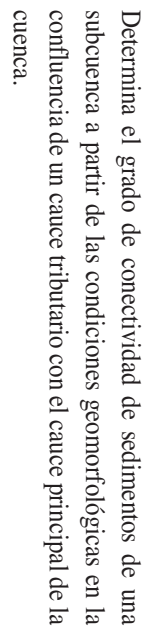 & 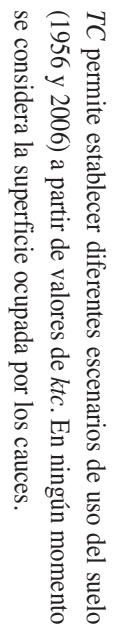 & 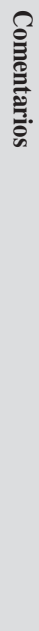 \\
\hline
\end{tabular}


representa la capacidad de transporte de sedimentos en cauces, y FC (Flow Conditions) son las condiciones de flujo de los cauces (continuo o efímero). El subíndice ' $a v$ ' en las variables representa la media del valor para la cuenca considerada y 'max' es el valor máximo de esta variable dentro de la cuenca y entre los escenarios considerados (1956 y 2006).

La tabla 1 muestra la clasificación de los factores citados, clasificados según los diferentes elementos paisajísticos diferenciados (ladera, cauce y subcuenca). El producto de dichos factores normalizados (para el período 1956-2006), determina los valores de $C C I$ o conectividad de sedimentos a escala de cuenca. El rango de valores de cada factor oscila entre 0 y 1 , para una menor o mayor conectividad, respectivamente. Se pretende que el índice desarrollado $(C C I)$ sea sensible a la evolución temporal de la conectividad sedimentológica de una cuenca para poder ser utilizado como una herramienta de análisis en la dinámica fluvial ante cambios y/o impactos en el área de drenaje y cauces.

Con el fin de desarrollar un análisis espacio-temporal del CCI y valorar el impacto de algunas actuaciones (construcción de diques), se ha aplicado el índice a tres escenarios diferentes: i) escenario de usos de suelo en 1956, considerado el escenario de referencia de conectividad; ii) escenario de usos de suelo en 2006 con presencia de diques; y iii) escenario hipotético de usos de suelo en 2006 sin presencia de diques. De este modo se pueden evaluar los cambios espaciales y temporales de la conectividad de sedimentos a escala de cuenca a partir del análisis de la evolución de los factores componentes del CCI. Asimismo, estos escenarios muestran la capacidad de (des)conexión de cada uno de los elementos participantes. Los escenarios de uso de suelo del 1956 y del 2006 se han cartografiado basados en fotografía área del vuelo USAF para el escenario de 1956 (resolución espacial 1m), mientras que para 2006 se ha empleado una imagen aérea del plan PNOA (Plan Nacional de Ortofotografía Aérea), con una resolución espacial de $0.5 \mathrm{~m}$.

A continuación se definen los distintos factores implicados en la conectividad total de sedimentos a escala de cuenca, detallando el método de cálculo de los mismos a partir de algebra de mapas con el programa SIG IDRISI (Eastman, 2003).

\subsection{Capacidad de transporte en laderas (TC)}

El modelo de erosión del suelo y transporte de sedimentos WaTEM-SEDEM permite la estimación de la capacidad de transporte de sedimentos media anual en laderas a partir del cálculo distribuido según:

$$
T C=k t c \cdot R \cdot K \cdot A^{1.4} \cdot S^{1.4}
$$

donde $R$ y $K$ son los factores de la RUSLE que determinan la erosividad de la lluvia y la erodibilidad del suelo, respectivamente; $A$ es el área de contribución, definida como la superficie que drena a un punto; y $S$ la pendiente del terreno. Verstraeten et al. (2007) demuestran la validez de este método para zonas semiáridas en las que la erosión por 
cárcavas y surcos es importante, frente a ecuaciones similares en las que no se tiene en cuenta este tipo de procesos.

Ya que los usos del suelo representan un factor muy importante en la conectividad de sedimentos a través de las laderas, el parámetro ktc es el responsable de expresar sus efectos, que se define a partir de la combinación de ktc-high y ktc-low (para superficies poco vegetadas y bien vegetadas, respectivamente). La combinación de los valores de $k t c$ se ha realizado a partir de la reclasificación de los valores del factor $C$ de la RUSLE, obteniendo finalmente un mapa de valores de ktc según la distribución de usos de $C$ para cada escenario. Borselli et al. (2008), sugiere la adaptación de parámetros con origen en otros modelos como el factor $C$, aplicándolo a nuevas aproximaciones de análisis de conectividad en cuencas. De acuerdo con esto, se asume una alta capacidad de transporte para valores de $C$ superiores a 0.20 siendo la capacidad de transporte baja por debajo de este umbral. Los valores de ktc deben estar calibrados por lo que se asumen como valores óptimos los obtenidos en trabajos anteriores donde se calibró el modelo WaTEMSEDEM, optimizando los valores ktc $\left(\right.$ ktc-low $=2 \cdot 10^{-6} ;$ ktc-high $\left.=2 \cdot 10^{-5}\right)($ Boix-Fayos et al., 2008; Quiñonero et al., en revisión).

\subsection{Conexión entre tributarios y cauce principal: factor geomorfológico (GF)}

Ambroise (2004) define como áreas contributivas a aquellas que están conectadas directamente a los cauces, generando un aumento de la escorrentía superficial y el volumen de sedimentos transportados. De este modo, aquellas subcuencas que se encuentran conectadas directamente al cauce principal a través de su propio cauce (tributario) son áreas contributivas y su conectividad es mayor que aquellas subcuencas no contributivas, que se conectan parcialmente, o no se conectan, con el cauce principal, debido a la presencia de superficies buffer.

La identificación e interpretación de los puntos de conexión entre cauces tributarios y el cauce principal (Fig. 2) es un aspecto clave en la conectividad de cuencas. El grado de conexión entre los cauces de la red de drenaje es un indicador efectivo de las condiciones de la cuenca tributaria y la respuesta en el punto de unión entre ambos, dando a conocer al mismo tiempo la relación existente con las partes del sistema aguas abajo. Adaptando y simplificando las metodologías propuestas por Fryirs et al. (2007a, b) y Borselli et al. (2008), para establecer la conectividad de cuencas, basadas en la identificación de formas de acumulación de sedimentos (buffers) de origen natural (llanura de inundación del cauce principal, conos aluviales, áreas con escasa pendiente), o aquellas derivadas de la alteración antrópica (nivelación de zonas agrícolas ocupando y rellenando cauces efímeros), que impiden la transferencia de sedimentos entre tributarios y el cauce principal, se determina la conexión de la red de drenaje desde una perspectiva geomorfológica (Fig. 2). Se ha establecido una clasificación simple de subcuencas conectadas por sus cauces (valor $=1$ ) y subcuencas no conectadas (valor $=0.5$ ), a partir de la red de drenaje principal, y según las observaciones derivadas del trabajo de campo y la fotointerpretación de fotografías aéreas de 1956 y 2006, se ha evaluado este factor para dos escenarios (1956 y 2006) en 28 subcuencas naturales del Alto Taibilla (Fig. 3). 


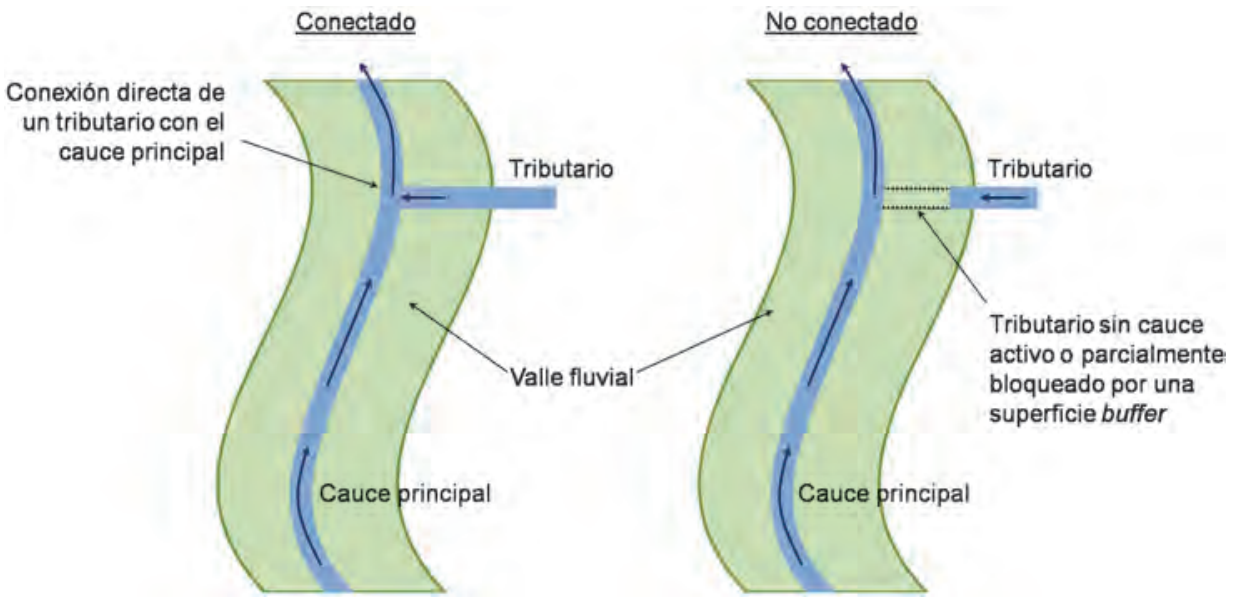

Figura 2. Clasificación del tipo de conexión entre tributarios y el cauce principal (redibujado a partir de Fryirs et al., 2007a).

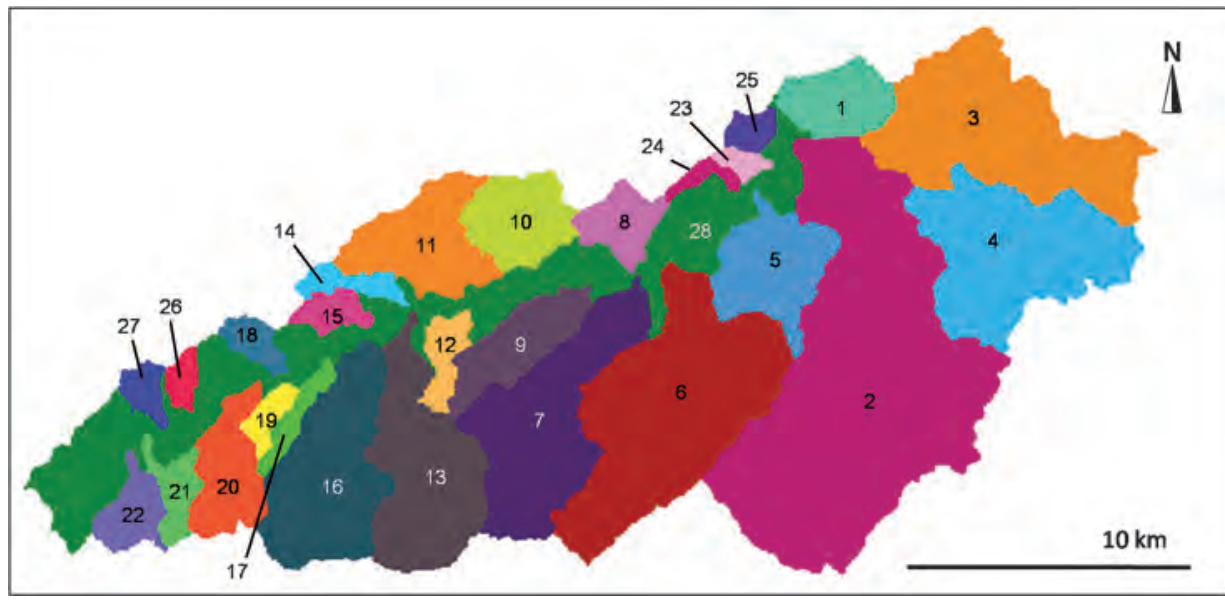

Figura 3. División en 28 subcuencas naturales de la cuenca del Alto Taibilla.

\subsection{Eficiencia de la retención de sedimentos de los diques (TE)}

Los elementos de origen artificial que pueden tener una elevada incidencia en la conectividad de los cauces del Alto Taibilla son los diques de retención de sedimentos. Su función e impacto se estima a partir de la eficiencia y capacidad de retención de sedimentos $(T E)$. La capacidad de retención de sedimentos de los diques también depende de las características y superficie vertiente al dique que determina su eficiencia como trampa. Por ello, cada dique adquiere el papel de barrera para la subcuenca artificial situada aguas arriba del punto donde se localiza el dique, asumiendo que su impacto no se limi- 
ta exclusivamente al cauce, sino a la transferencia de sedimentos a través del cauce de toda la superficie que vierte al dique (López-Vicente et al., 2011). De este modo se ha establecido una clasificación del efecto de la conectividad derivado de los diques a partir de su TE para el escenario 2006 con 94 diques, y asumiendo que el mayor grado de conectividad se asocia a aquellas subcuencas y escenarios en las que no existen diques. Esta ecuación calcula $T E$ en función de la capacidad de almacenamiento de un dique y el área vertiente a dicho dique. La capacidad de retención (TE) de los diques de retención de sedimentos se ha calculado según la ecuación de Brown (1943). Debido a que los diques sufren un proceso gradual de colmatación y por ello pierden capacidad de almacenamiento, se han calculado dos valores de TE para cada dique de retención de sedimentos; el primer valor se basa en el volumen original del dique, mientras que el segundo valor toma como referencia el volumen estimado a partir de las mediciones de campo realizadas. Finalmente, se ha empleado el valor medio de $T E$ para calcular la exportación de sedimentos basados en los volúmenes de sedimentos medidos en el campo.

\subsection{Cauces de caudal continuo y cauces efímeros: factor de condiciones de flujo (FC)}

Hemos clasificado la red de drenaje del Alto Taibilla en dos tipos de cauces según sus condiciones de flujo: cauces con caudal continuo y efímero (Fig. 4). Aunque durante eventos de precipitación mayores las descargas totales de sedimentos tienden a ser mucho mayores en cauces con carácter efímero que en cauces con caudales permanentes (Hooke, 2006), la conectividad de los sedimentos será mayor en aquellos cauces que presentan un caudal continuo, ya que la intermitencia de caudales en cauces de carácter efímero se debe a la frecuencia con que se produce un evento lluvioso de magnitud suficiente para producir la escorrentía capaz de transportar sedimentos a lo largo de los cauces. De este modo, los cauces efímeros responden a un carácter episódico de la exportación de los sedimentos (Lana-Renault, 2011) y, por tanto, la capacidad y el tiempo de almacenamiento de sedimentos en los cauces son mucho mayores en cauces efímeros, mientras que aquellos que poseen un caudal permanente realizan una evacuación constante que dificulta la sedimentación. De este modo, en la tabla 1 se establece una clasificación determinada por las condiciones de flujo dominantes (cauces con caudal continuo $=1$; cauces efímeros $=0.5)$, observadas en el campo.

\subsection{Transporte de sedimentos en cauces $(\mathrm{SP})$}

Para calcular el transporte de sedimentos en los cauces se ha optado por la fórmula original del cálculo de la capacidad de transporte en cauces (Dietrich et al., 2003), utilizando la ecuación:

$$
q_{s}=k \cdot Q^{m} \cdot S^{n}
$$

donde $Q$ es el caudal por ancho de sección, $k$ una constante de proporcionalidad del caudal, y $S$ la pendiente local. El carácter efímero y la falta de series de datos de caudales, debido a la ausencia de estaciones hidrológicas en la gran mayoría de los cauces de la cuenca de estudio, hace que se opte por sustituir $Q$, por el área de contribución $(A)$, ya 

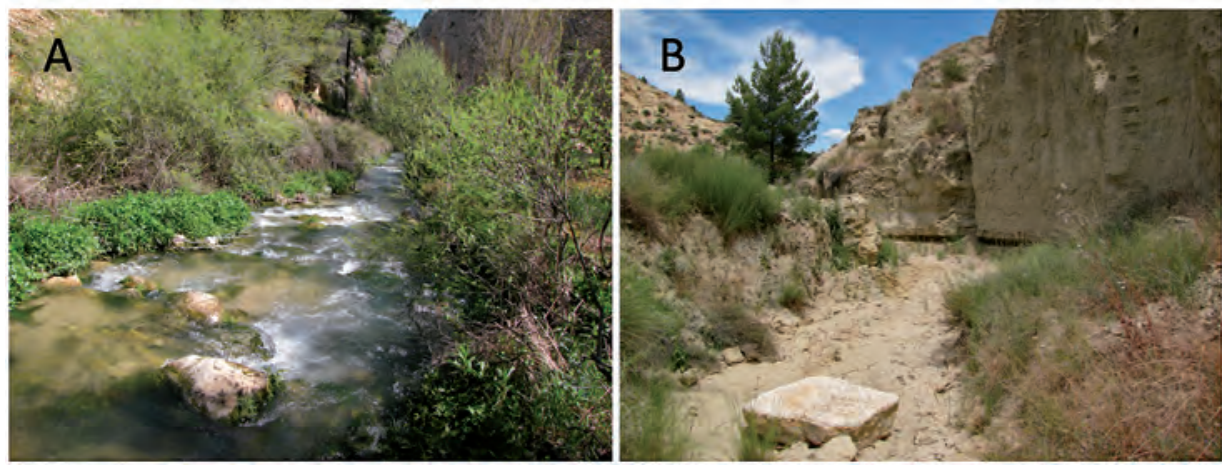

Figura 4. Tipología de cauces según sus condiciones de flujo: A) tramo de cauce con caudal continuo en el río Taibilla; y B) tramo de cauce de caudal efímero en el cauce principal de un tributario.

que la concentración de flujo que determina la formación de cauces está en función del área de drenaje (Dietrich et al., 2003), siendo ésta una variable de gran importancia en estudios de conectividad de sedimentos (Beven y Wood, 1983; Ambroise, 2004; Fryirs et al., 2005; 2007a, b; Borselli et al., 2008). Además, las relaciones erosión-escorrentía no son lineales, pero si responden a una dependencia de la escala espacial (López-Vicente et al., 2011), ya que la exportación de sedimentos depende de la extensión y localización de las áreas fuente de sedimentos (Walling, 1983).

Las constantes $m$ y $n$ adoptan el valor de 1.4, tal y como proponen Prosser y Rustomji (2000), basándose en una extensa revisión de estudios experimentales. Finalmente, es posible calcular un escenario único de capacidad de transporte de sedimentos en cauces ( $S P=$ Stream Power) según la ecuación:

$$
S P=A^{1.4} \cdot S^{1.4}
$$

\section{Resultados}

La tabla 2 muestra los principales resultados de la aplicación del índice $(C C I)$ a los escenarios de uso de 1956, 2006 sin diques y 2006 con diques.

i) Capacidad de transporte de sedimentos en laderas: factor $T C$

La evolución de la capacidad de transporte en las laderas de la cuenca del Alto Taibilla entre 1956 y 2006, muestra cambios importantes con una reducción desde el valor medio de $T C$ de cuenca de 0.335 en 1956 hasta 0.085 en 2006 (Tabla 2) (reducción media del 75\%). La reducción drástica de los valores de TC se corresponde con los cambios de uso del suelo del Alto Taibilla durante el período 1956-2006 que evolucionaron hacia una mayor cobertura vegetal y protección del suelo, y por tanto de desconexión de los sedimentos desde las laderas a los cauces. 
ii) Capacidad de retención de sedimentos de los diques: factor $T E$

La superficie de cuenca vertiente del Alto Taibilla afectada por la construcción de diques es de $117.3 \mathrm{~km}^{2}$, lo que supone un $37.3 \%$ de la cuenca, ninguno de ellos localizado en el cauce principal del río Taibilla. La presencia de diques se limita a 5 subcuencas (1, 2, 6, 13 y 25). Los valores medios por subcuenca de $T E$ son en general bajos (media de las 5 subcuencas con diques de 37\%), aunque la variabilidad de valores específicos de $T E$ para el conjunto de los diques es grande, entre 5.2\% y $98 \%$. Por otra parte, el escenario de 1956 no presenta diques construidos dentro de la cuenca del Alto Taibilla, por lo que este factor no influye en la disminución de la conectividad en ese escenario.

iii) Conexión de subcuencas a partir de aspectos geomorfológicos: factor $G F$

Mientras que en el escenario de referencia (1956) se observan conexiones claras de la mayoría de los cauces tributarios con el río Taibilla, en el 2006 aparecen algunas subcuencas más desconectadas por barreras geomorfológicas (Tabla 2). Se produce un aumento de los buffers con efecto de desconexión y aumento de las áreas no contributivas, que pasan del $4.9 \%$ (5 subcuencas) al 7.7\% (8 subcuencas) de la superficie del Alto Taibilla entre 1956 y 2006. El principal factor la alteración de superficies es antrópico: nivelaciones para el aprovechamiento agrícola, utilizando zonas de acumulación sedimentaria (conos, abanicos aluviales) en los puntos de conexión entre tributarios y el cauce principal. Todas las subcuencas desconectadas en 2006 se localizan en el sector oeste de la cuenca, en la zona de cabecera.

iv) Conectividad en cauces: factores $F C$ y $S P$

La capacidad de transporte de sedimentos en los cauces muestra valores máximos de $S P$ en zonas de cabecera donde las pendientes son mayores a lo largo de los cauces principales. Los valores de $S P$ normalizado oscilan entre 0.21 y 1 y no muestran diferenciación temporal entre escenarios pues sólo se dispone de información actual sobre el mismo.

A esto hay que añadir que la mayor parte de los cauces de la red de drenaje tienen un carácter efímero por lo que la conectividad se ve reducida de manera significativa, ya que no existe un caudal continuo capaz de movilizar los sedimentos de manera constante. El total de la red de drenaje suma $231 \mathrm{~km}$ de longitud de cauces, de los que $176 \mathrm{~km}$ se han identificado como cauces de carácter efímero y $55 \mathrm{~km}$ presentan un caudal continuo.

\subsection{Conectividad de sedimentos a escala de cuenca bajo diferentes escenarios de uso del suelo: Índice de Conectividad de Cuenca (CCI)}

Los resultados obtenidos de $C C I$ basados en valores medios por subcuencas indican que la cuenca del Alto Taibilla pasó de tener una moderada conectividad en $1956(0.12)$ a una baja conectividad con los usos de suelo de 2006, incluyendo los diques de retención de sedimentos (0.029). Esto representa una reducción significativa estadísticamente (Tabla 2) de un 76\% entre 1956 y 2006 (Tabla 2; Fig. 5, 6 y 7). El mayor grado de conec- 


\begin{tabular}{|c|c|c|c|c|c|c|c|c|c|c|c|c|c|c|c|c|c|c|c|c|}
\hline \multirow{7}{*}{ 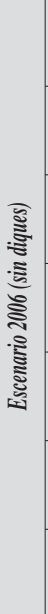 } & 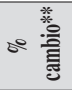 & శ్ర & t & $\bar{\sigma}$ & $\stackrel{2}{2}$ & ప & 8 & $\approx$ & 8 & $\infty$ & $\approx$ & 5 & ஓ & 5 & $\infty$ & $\cong$ & 8 & 2 & $\stackrel{\infty}{\infty}$ & $\mathbb{N}$ \\
\hline & ت্ర & 范 & $\stackrel{\overbrace{}}{0}$ & ळ̈ & 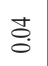 & 营 & $\tilde{\Xi}$ & 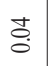 & 苞 & $\stackrel{\wp}{0}$ & $\stackrel{0}{0}$ & $\stackrel{\vec{\sigma}}{\circ}$ & $\bar{\sigma}$ & $\stackrel{0}{\circ}$ & $\stackrel{8}{8}$ & $\bar{\sigma}$ & $\stackrel{\circ}{0}$ & $\stackrel{\partial}{\sigma}$ & $\stackrel{\text { ŏ }}{\circ}$ & $\stackrel{\overbrace{}}{0}$ \\
\hline & 5 & ڤू & : & & $\begin{array}{l}\infty \\
\stackrel{\infty}{0} \\
0\end{array}$ & $\underset{\substack{+0}}{\stackrel{0}{0}}$ & : & $\vec{\sigma}$ & مิ & ڤั) & مू & مิ & ํ. & مू & 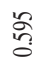 & 응 & $\begin{array}{l}\text { مे } \\
\text { مे }\end{array}$ & $\begin{array}{l}\text { 응 } \\
\text { an }\end{array}$ & 을 & $\begin{array}{l}\text { 응 } \\
\text { है }\end{array}$ \\
\hline & Б) & to & 离 & $\frac{0}{3}$ & $\stackrel{8}{8}$ & శ్రి: & Fै & $\stackrel{\circ}{0}$ & Бे. & 守 & $\underset{\Xi}{\rightleftarrows}$ & 芯 & 志 & $\frac{\pi}{50}$ & $\stackrel{\overbrace{}}{\widehat{s}}$ & 宮 & 莽 & $\begin{array}{l}\widetilde{\alpha} \\
\text { ळ. }\end{array}$ & 공 & \&. \\
\hline & D & - & - & - & - & - & - & - & - & - & - & - & 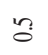 & - & $n$ & $n$ & - & $n$ & - & $n$ \\
\hline & $\Leftrightarrow$ & 0 & 0 & 0 & 0 & 0 & 0 & 0 & 0 & 0 & 0 & 0 & 0 & 0 & 0 & 0 & 0 & 0 & 0 & 0 \\
\hline & 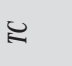 & $\frac{\mathscr{P}}{0}$ & $\begin{array}{l}\infty \\
\stackrel{0}{0}\end{array}$ & 莺 & 总 & $\underset{\stackrel{\infty}{\circ}}{\stackrel{\infty}{0}}$ & ஜ̊. & $\begin{array}{l}\infty \\
\stackrel{0}{0} \\
0\end{array}$ & $\frac{\sigma}{0}$ & 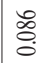 & $\frac{0}{0}$ & $\underset{\delta}{\infty}$ & $\frac{0}{5}$ & $\frac{\text { 专 }}{0}$ & $\overrightarrow{\mathrm{g}}$ & $\overrightarrow{5}$ & $\frac{\infty}{0}$ & \%) & 苛 & $\stackrel{\infty}{\circ}$ \\
\hline \multirow{7}{*}{ 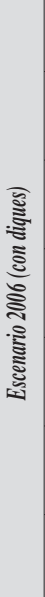 } & ¿。 & 2 & $尺$ & $\bar{\sigma}$ & 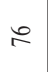 & ఫ & 尺 & 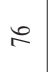 & 8 & 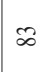 & 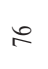 & 5 & வे & \$ & $\infty$ & $\approx$ & 8 & 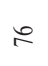 & $\infty$ & $\mathbb{N}$ \\
\hline & 히 & 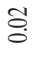 & $\stackrel{0}{0}$ & $\stackrel{\circ}{\circ}$ & $\underset{0}{0}$ & $\stackrel{t}{0}$ & ฮ̊. & $\stackrel{\Delta}{0}$ & $\stackrel{t}{0}$ & ชூo. & $\stackrel{0}{0}$ & $\vec{\circ}$ & $\bar{\sigma}$ & $\stackrel{\tilde{c}}{0}$ & $\stackrel{8}{0}$ & है. & $\stackrel{2}{\circ}$ & $\overrightarrow{0}$ & ठ̊. & ¿̊. \\
\hline & 证 & 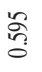 & 영 & 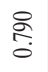 & $\begin{array}{l}\infty \\
\infty \\
0 \\
0\end{array}$ & $\begin{array}{l}\text { t⿱艹o } \\
\stackrel{0}{0}\end{array}$ & 象 & $\vec{\sigma}$ & $\begin{array}{l}\text { 응 } \\
\text { an }\end{array}$ & 응 & $\begin{array}{l}\text { 总 } \\
\text { है }\end{array}$ & $\begin{array}{l}\text { 哭 } \\
\text { م. }\end{array}$ & $\begin{array}{l}\text { 合 } \\
\text { م. }\end{array}$ & $\begin{array}{l}\text { 合 } \\
\text { - }\end{array}$ & $\begin{array}{l}\text { مू } \\
\text { م̂े }\end{array}$ & 蓇 & $\begin{array}{l}\text { مू } \\
\text { مे }\end{array}$ & $\begin{array}{l}\text { 哭 } \\
\text { م. }\end{array}$ & 䓂 & 응 \\
\hline & ఓ & 矛 & $\begin{array}{l}\text { 今్ } \\
\text { ลे }\end{array}$ & $\frac{0}{3}$ & $\underset{8}{\stackrel{8}{0}}$ & శ్రి: & $\begin{array}{l}\mathcal{F} \\
\stackrel{0}{0}\end{array}$ & $\begin{array}{l}\mathscr{0} \\
\stackrel{0}{0}\end{array}$ & बे. & 孚 & 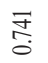 & 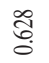 & 芯 & $\underset{0}{\mathbb{d}}$ & 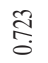 & \begin{tabular}{l}
$\mathscr{D}$ \\
$:$ \\
\hdashline
\end{tabular} & 夺 & $\begin{array}{l}\text { ऽે } \\
\text { o. }\end{array}$ & $\underset{\mathbb{S}}{\vec{\delta}}$ & $\underset{0}{8}$ \\
\hline & 必 & - & - & - & - & - & - & - & - & - & - & - & $n$ & - & $n$ & $n$ & - & $n$ & - & $n$ \\
\hline & $\Leftrightarrow$ & $\begin{array}{l}\tilde{n} \\
\text { â }\end{array}$ & $\stackrel{2}{0}$ & $\stackrel{8}{8}$ & $\stackrel{8}{0}$ & $\stackrel{8}{8}$ & $\stackrel{\overbrace{}}{0}$ & $\underset{0}{8}$ & $\underset{0}{8}$ & 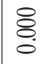 & \&ి. & \&̊. & \&̊. & \begin{tabular}{l}
\multirow{2}{*}{} \\
है
\end{tabular} & 官 & \&ి. & ๕ి & \&్. & §ి. & : \\
\hline & $\leftleftarrows$ & $\frac{\tilde{F}}{8}$ & $\begin{array}{l}\infty \\
\stackrel{0}{\circ}\end{array}$ & 蒿 & î. & $\stackrel{\infty}{\circ}$ & $\begin{array}{l}\infty \\
\stackrel{0}{0} \\
\stackrel{0}{0}\end{array}$ & $\begin{array}{l}\infty \\
\stackrel{\leftrightarrow}{0} \\
0\end{array}$ & ळे & 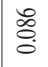 & $\frac{0}{0}$ & $\begin{array}{l}\infty \\
\stackrel{\delta}{0}\end{array}$ & 壱 & $\frac{J}{0}$ & $\overline{\widetilde{\delta}}$ & $\vec{\circ}$ & $\stackrel{\infty}{\circ}$ & 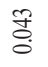 & 苂 & $\stackrel{\infty}{0}$ \\
\hline \multirow{7}{*}{ 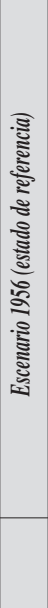 } & 히 & $\frac{O}{0}$ & $\stackrel{8}{0}$ & $\stackrel{8}{0}$ & $\frac{n}{0}$ & $\stackrel{\text { ڤ̆ }}{0}$ & so. & 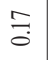 & $\stackrel{ }{0}$ & $\frac{n}{0}$ & $\stackrel{\text { ¿̊ }}{0}$ & to. & $\stackrel{ \pm}{0}$ & 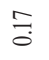 & $\stackrel{\overbrace{}}{\circ}$ & $\stackrel{t}{\sigma}$ & $\stackrel{\square}{0}$ & $\stackrel{\leftrightarrow}{\circ}$ & ț & $\stackrel{\circ}{0}$ \\
\hline & 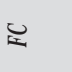 & 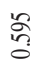 & : & $\stackrel{2}{2}$ & $\begin{array}{l}\infty \\
\infty \\
\infty \\
0\end{array}$ & $\begin{array}{l}\text { t⿱艹 } \\
\stackrel{0}{0} \\
0\end{array}$ & : & $\vec{\sigma}$ & 咢 & 瓷 & 咢 & 哭 & 蓇 & 咢 & 哭 & 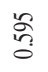 & 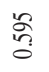 & 咢 & 誉 & 总 \\
\hline & ह & so & 苈 & $\frac{0}{\sqrt[n]{3}}$ & $\stackrel{8}{8}$ & శ్రి: & శ్t & $\underset{0}{:}$ & $\overrightarrow{\mathrm{b}}$ & 守 & $\underset{0}{\stackrel{F}{\delta}}$ & 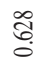 & 芯 & $\underset{0}{\tilde{\sigma}}$ & 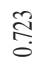 & \begin{tabular}{l}
$\infty$ \\
$\infty$ \\
\hdashline
\end{tabular} & 莳 & $\begin{array}{l}\widetilde{a} \\
\text { ळ. }\end{array}$ & $\underset{\mathbb{S}}{\vec{\Xi}}$ & $\stackrel{8}{8}$ \\
\hline & 头 & - & - & - & - & - & - & - & - & - & - & - & - & - & - & 3 & - & $n$ & - & $n$ \\
\hline & 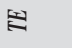 & 0 & 0 & 0 & 0 & 0 & 0 & 0 & 0 & 0 & 0 & 0 & 0 & 0 & 0 & 0 & 0 & 0 & 0 & 0 \\
\hline & $\leftleftarrows$ & $\underset{\tilde{\sigma}}{\tilde{\sigma}}$ & $\overrightarrow{\bar{I}}$ & ö & శ్రి & $\begin{array}{l}\text { ণ্ } \\
\text { f }\end{array}$ & $\frac{a}{0}$ & $\begin{array}{l}\stackrel{8}{0} \\
0 \\
0\end{array}$ & ते & 응 & $\begin{array}{l}\text { oे } \\
\stackrel{b}{0}\end{array}$ & $\stackrel{0}{0}$ & 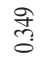 & ને & $\stackrel{\circ}{\circ}$ & 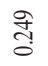 & $\begin{array}{l}\text { 就 } \\
\text {. }\end{array}$ & $\frac{\infty}{0}$ & $\frac{8}{0}$ & ठ્ণ \\
\hline & 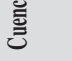 & 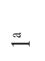 & $\tilde{\sim}$ & $m$ & $\nabla$ & in & है & $r$ & $\infty$ & $a$ & ㅇ & $=$ & 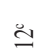 & $\tilde{m}$ & 守 & $\stackrel{1}{n}$ & $\underline{0}$ & $\stackrel{1}{ \pm}$ & $\infty$ & 2 \\
\hline
\end{tabular}




\begin{tabular}{|c|c|c|c|c|c|c|c|c|c|c|c|c|}
\hline 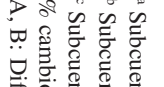 & 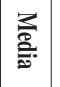 & $\mathbb{\infty}$ & $\frac{9}{7}$ & $\approx$ & 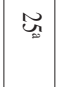 & $\tilde{\perp}$ & $\mathbb{\omega}$ & ్ㅠ & $\simeq$ & $\tilde{0}$ & E & \\
\hline 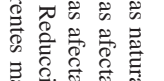 & $\begin{array}{l}0 \\
\dot{u} \\
\breve{s} \\
\end{array}$ & $\stackrel{ }{\stackrel{0}{\Xi}}$ & 응 & 故 & 엉 & $\dot{8}$ & 芯 & 沜 & : & 怘 & $\vec{\imath}$ & \\
\hline $\begin{array}{lll}\infty & \infty \\
0 & 0 & 0 \\
0 & 0 & 0\end{array}$ & $P$ & $\circ$ & 0 & 0 & $\circ$ & o & 0 & 0 & $\circ$ & 0 & 주 & 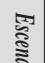 \\
\hline 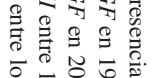 & $\stackrel{8}{8}$ & - & in & - & - & - & - & - & - & in & જ) & ๘ั \\
\hline 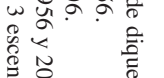 & $\stackrel{\circ}{0}$ & $\begin{array}{l}\circ \\
\text { 龺 }\end{array}$ & 产 & : & 으= & $\begin{array}{l}\stackrel{\circ}{\infty} \\
\vdots \\
\sigma\end{array}$ & 总 & $\begin{array}{l}\infty \\
\substack{+\infty \\
\infty}\end{array}$ & $\underset{\perp}{\stackrel{+}{\infty}}$ & $\begin{array}{l}\stackrel{P}{\infty} \\
\dot{\infty} \\
u\end{array}$ & $\succcurlyeq$ & 产 \\
\hline * & 高 & $\overline{8}$ & 宮 & 足 & O & 宮 & 兽 & Ö. & 柋 & $\begin{array}{l}0 \\
\dot{厶} \\
0 \\
0\end{array}$ & $\vec{\partial}$ & ఏ. \\
\hline 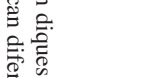 & 임 & $\ddot{\infty}$ & 은 & 음 & 总 & f & $\stackrel{0}{u}$ & ○ & 언 & 怘 & $\widehat{\Omega}$ & \\
\hline 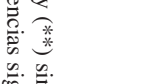 & 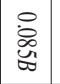 & : & : & $\stackrel{\circ}{\stackrel{⿹}{-}}$ & $\stackrel{\circ}{\stackrel{9}{+}}$ & : & 京 & 离 & : & 总 & $\vec{\imath}$ & \\
\hline 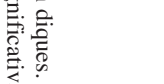 & 离 & вे & $\ddot{8}$ & 8े & 志 & $\ddot{8}$ & 8 & \&े & 8 & Oें & त्र & \\
\hline 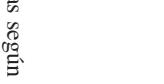 & 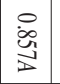 & - & in & - & - & - & - & in & - & i & ક각 & 冡 \\
\hline & $\stackrel{\circ}{\dot{\omega}}$ & $\begin{array}{l}\circ \\
\text { 龺 }\end{array}$ & 产 & 용 & 으= & $\begin{array}{l}\circ \\
\infty\end{array}$ & 怘 & $\begin{array}{l}0 \\
\substack{+\infty \\
\infty}\end{array}$ & $\begin{array}{c}\stackrel{0}{+} \\
\stackrel{+}{\infty} \\
\infty\end{array}$ & $\begin{array}{l}\stackrel{\odot}{\infty} \\
\underset{u}{*}\end{array}$ & $y$ & $\frac{5}{3}$ \\
\hline & 宰 & $\overline{8}$ & 唐 & 范 & 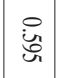 & 芦 & : & 芯 & 范 & 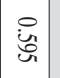 & $\overrightarrow{\mathrm{D}}$ & 㧣 \\
\hline & $\begin{array}{l}\stackrel{\circ}{0} \\
\text { 总 } \\
\end{array}$ & ¿े & в & 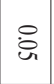 & $\stackrel{\circ}{\circ}$ & $\stackrel{\circ}{\dot{\omega}}$ & $\stackrel{\circ}{\circ}$ & $\cong$ & 竎 & 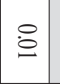 & 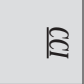 & \\
\hline & 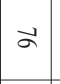 & $\widehat{a}$ & $\stackrel{\infty}{\sim}$ & 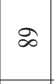 & $\overrightarrow{0}$ & $\vec{\mp}$ & $\approx$ & is & $\infty$ & ખు & $\frac{0}{3}$ & \\
\hline & 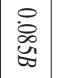 & : & 응 & $\begin{array}{l}\stackrel{0}{0} \\
= \\
\end{array}$ & $\stackrel{\circ}{\stackrel{亠}{+}}$ & $\begin{array}{l}\text { 谂 } \\
\text {. }\end{array}$ & 京 & 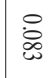 & $\stackrel{\circ}{\circ}$ & 离 & $\vec{\imath}$ & \\
\hline & 8 & 0 & 0 & 0 & 0 & 0 & 0 & 0 & 0 & 0 & त्न & \\
\hline & 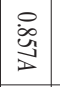 & - & 은 & - & - & - & - & in & - & in & בె & 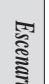 \\
\hline & $\stackrel{\circ}{\dot{\omega}}$ & $\begin{array}{l}\circ \\
\text { 龺 }\end{array}$ & 产 & के & 으= & $\begin{array}{l}\circ \\
\infty \\
\sigma\end{array}$ & 总 & 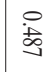 & 古 & $\begin{array}{l}\stackrel{0}{\infty} \\
\underset{u}{*}\end{array}$ & 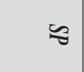 & క్ \\
\hline & 吕 & $\dot{\overline{8}}$ & 范 & 范 & 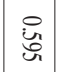 & 怘 & 惫 & 岕 & 它 & 家 & 今 & है: \\
\hline & $\begin{array}{l}\stackrel{8}{0} \\
\stackrel{\Xi}{\varpi}\end{array}$ & : & 8 & 号 & $\stackrel{\circ}{\circ}$ & $\stackrel{\circ}{\omega}$ & 咅 & $\cong$ & : & 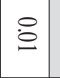 & $\widehat{E}$ & \\
\hline & $\vec{\omega}$ & 9 & $\stackrel{\infty}{=}$ & $\infty$ & ని & $\vec{F}$ & $\approx$ & i & $\infty$ & ખ) & 鱼 & \\
\hline
\end{tabular}




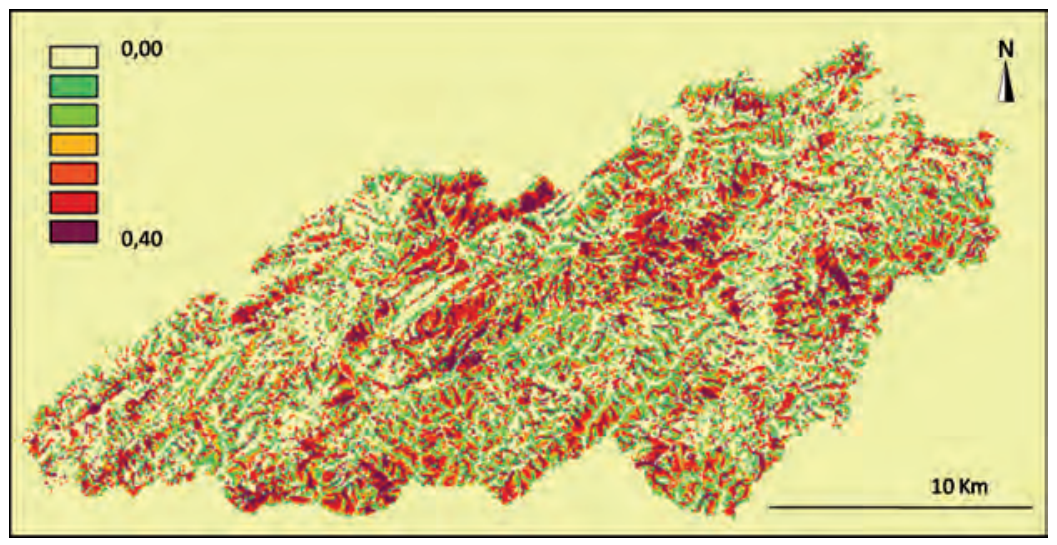

Figura 5. Escenario ICC de referencia para 1956: valores por píxel (resolución de $30 \mathrm{~m}$ ).

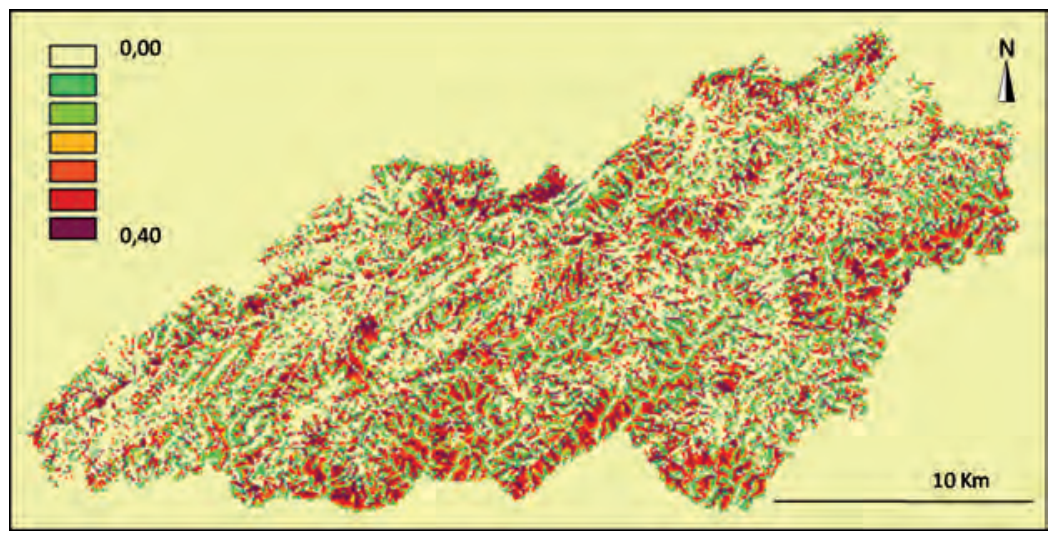

Figura 6. Escenario ICC para 2006 con diques: valores por píxel (resolución de 30 m).

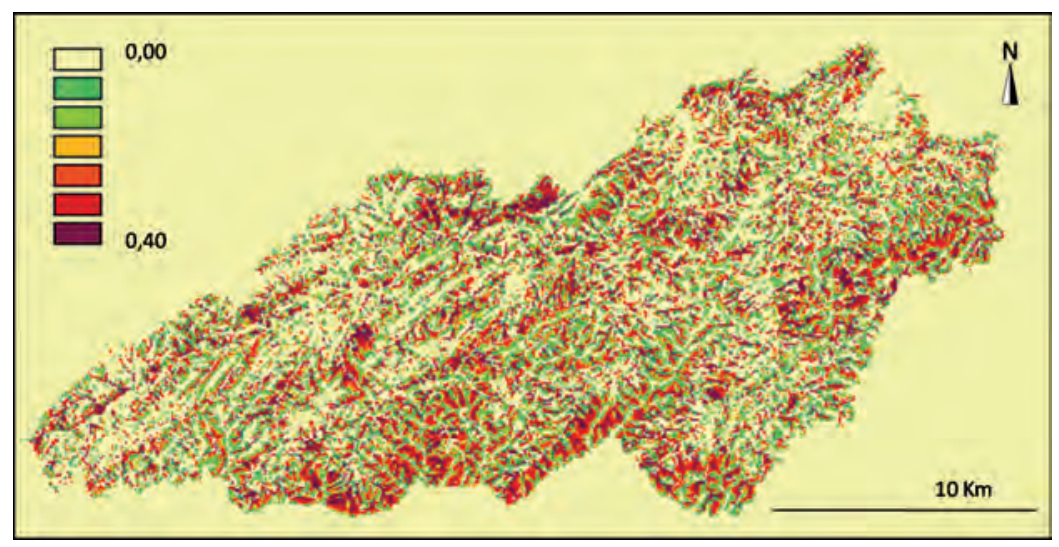

Figura 7. Escenario ICC para 2006 sin diques: valores por píxel (resolución de 30 m). 
tividad dentro de la cuenca se localiza en las proximidades de los cauces, especialmente en sectores de cabecera (Fig. 5 y 6). La conectividad total de cuenca se ve reducida significativamente entre 1956 y 2006 en todas las subcuencas de estudio debido principalmente a la reducción de $T C$, y en segundo lugar debido a la construcción de diques (Tabla 2). Analizando los valores a escala de subcuenca, la variabilidad espacial del CCI oscila entre un descenso de la conectividad de sedimentos del $61 \%$ para la subcuenca de Arroyo Tercero (cuenca 3) hasta una reducción máxima del $92 \%$ para la subcuenca 22 (Fig. 3, sector Oeste en la cabecera de la cuenca).

En la simulación de un hipotético escenario de conectividad de sedimentos en el 2006 sin la presencia de diques (Fig. 7) la reducción de CCI es de $73 \%$ respecto a 1956 , por lo que la conectividad media es ligeramente superior (3\%) al escenario real con diques en 2006 (Tabla 2).

\section{Discusión}

\subsection{Factores y aproximación a la conectividad de sedimentos a escala de cuenca}

El método propuesto del CCI, basado en trabajo de campo, fotointerpretación y modelización SIG, ha permitido analizar la evolución de la conectividad desde una perspectiva espacio-temporal de cuenca a partir de los cambios de usos del suelo, medidas de conservación del suelo y factores geomorfológicos. Además, también ofrece la oportunidad de distinguir la conectividad individual de los elementos que componen el sistema fluvial según una combinación de escalas espaciales. El método se ha desarrollado a partir de una revisión previa de métodos existentes e intentando combinar las fortalezas de varios de ellos. Por ejemplo, Borselli et al. (2008) propone un método similar al de este trabajo, que permite identificar cambios de la conectividad en áreas que han sufrido cambios geomorfológicos y alteraciones antrópicas importantes para eventos de precipitación en los Apeninos (Italia). Establece una relación entre el área fuente de sedimentos situada aguas arriba de un punto (ej.: dique, inicio de un cauce), y la distancia a un sumidero local (ej.: cauce principal) donde se acumulan los sedimentos transportados. Para ello, Borselli et al. (2008) distinguen el IC (Index of Connectivity), a partir de modelización SIG, y el FIC (Field Index of Connectivity), basado en observaciones de campo y que valida los resultados de $I C$. Pero la correspondencia entre ambos índices, es sólo válida localmente, ya que la magnitud y frecuencia de los eventos tormentosos juegan un papel importante en la evaluación de la conectividad en zonas semiáridas. Pequeños cambios en el patrón de precipitaciones pueden dar lugar a un mayor aporte de sedimentos desde laderas hasta los cauces, provocando importantes cambios morfológicos en los mismos (Coppus y Imeson, 2002; Hancock y Coulthard, 2012). No obstante, Lopez-Vicente et al. (2011) emplean el método de Borselli et al. (2008) en zonas de los Pirineos (España), estableciendo una valoración a escala temporal con el planteamiento de una serie de escenarios de evolución de los usos de suelo y obteniendo interesantes y contrastadas conclusiones a escala de cuenca. 
Desde nuestro punto de vista, uno de los aspectos clave en la conectividad de una cuenca es la relación ladera-cauce. La relativa complejidad de algunos métodos como el propuesto por Lane et al. (2004) y desarrollado para predecir el riesgo de conectividad según la probabilidad que un punto del paisaje esté conectado a la red de drenaje con el fin de priorizar y optimizar decisiones de gestión territorial; o el desarrollo del modelo hidrológico Coup2D, ideado por Michaelides y Wainwright (2002) que establece una relación entre la respuesta hidrológica de cuencas a partir de la morfología entre laderas y cauce (uniendo conceptos de buffering y coupling), contrasta con el método que desarrolla Hooke (2003) y que también aplica Boix-Fayos et al. (2007) basado en observaciones y cartografía directa en el campo de las conexiones ladera-cauce a partir de los flujos de sedimentos que se identifican entre ambos. Otro ejemplo es el estudio de la eficiencia del aporte de sedimentos entre laderas y cauces de McHugh et al. (2002), que resulta ser una propuesta viable de gran interés y utilidad para la gestión de cuencas (Bracken y Croke et al., 2007) utilizando hipótesis sencillas y valores medios de producción de sedimentos.

Por último, el índice propuesto en este trabajo $(C C I)$ intenta profundizar en los aspectos geomorfológicos y basa su factor $G F$ en los conceptos y métodos de Fryirs et al. (2007a, b) y Faulkner et al. (2008), que advierten de la gran capacidad de algunos elementos geomorfológicos dentro de la cuenca que facilitan la discontinuidad de flujos de agua y sedimentos, tales como depósitos de sedimentos en laderas, abanicos aluviales y piedemontes (buffers y barreras). Sin embargo, estos métodos citados ofrecen perspectivas globales de conectividad de cuenca con carácter estático en las que no se definen con detalle las relaciones de conexión entre los diferentes elementos de la cuenca y no introducen factores más dinámicos temporalmente como cambios de uso del suelo y reajustes morfológicos de cauces. Borselli et al. (2008) también introduce un factor geomorfológico, sin embargo, y a pesar de utilizarlo como unidad dentro de la cuenca, la perspectiva sigue siendo local, sin que sus efectos se puedan identificar a una escala más amplia (cuenca/regional). El CCI se plantea como un método de sencilla aplicación que combina diferentes escalas espaciales y escenarios temporales, basándose en la evaluación de distintos factores topográficos, antrópicos, hidrológicos y geomorfológicos, lo que lo hace versátil y funcional en un marco de gestión territorial. Además, puede diferenciar entre elementos de la cuenca y evaluar sus cambios en conectividad de forma independiente.

\subsection{Cambios temporales en la conectividad: elementos de (des)conectividad}

La evolución de los usos del suelo en la cuenca del Alto Taibilla muestra superficies más protegidas en gran parte de la cuenca por los efectos de la revegetación natural o resultado de reforestaciones (de Vente et al., 2007), pero también la concentración de la actividad agrícola junto a los cauces, lo que confiere un mayor grado de conectividad ladera-cauce. A pesar de ello, el efecto global de los cambios de uso del suelo es de desconexión y reducción de la conectividad, debido principalmente a una gran reducción de los valores de $T C$ entre 1956 y 2006 (Tabla 2). 
La comparación de escenarios en 2006 con diques y sin diques, permite establecer el impacto de la construcción de la red de diques de retención de sedimentos. Los diques pueden ejercer un papel muy importante localmente, e incluso a escala de subcuenca, en la reducción de la conectividad de sedimentos, pero su impacto dentro de la cuenca del Alto Taibilla no es tan significativo como el efecto de los cambios de uso del suelo. Mientras el conjunto de las subcuencas con presencia de diques $(1,2,6,13$ y 25) muestran una reducción media del $13 \pm 7 \%$ por causa de los diques en 2006 , este efecto se reduce a tan sólo el 3\% para toda la cuenca del Alto Taibilla. Por otro lado es importante destacar que un cambio en la conectividad en cauces puede tener un mayor impacto sobre la exportación de sedimentos que un cambio en la conectividad en laderas, ya que a través de los cauces circulan mayores volúmenes de sedimentos. Los efectos del factor $G F$ implican un $6 \%$ de reducción de la conectividad entre 1956 y 2006, por barreras geomorfológicas en las conexiones entre cauces. Quizá en condiciones de mayor entrada de sedimento al cauce puede aumentar el peso de este factor; en este caso hay una evolución en el período estudiado a menos entrada de sedimento desde las laderas al cauce por el crecimiento de la cobertura vegetal. Por otra parte, las condiciones predominantemente efímeras de la red de cauces principales del Alto Taibilla favorecen que los cauces actúen como sumideros temporales de los sedimentos transferidos desde las laderas (Gallart et al. 2005), aunque actualmente hay una gran reducción de aportes sedimentarios de las laderas con respecto a 1956. La capacidad de evacuación de sedimentos de estos cauces efímeros en zonas semiáridas depende, pues, de que se produzca un evento lluvioso de magnitud e intensidad tales que genere una escorrentía capaz de movilizar los sedimentos acumulados en el lecho de los cauces (Hooke, 2003). Sin embargo, aunque esto afecta a la mayor parte de los tributarios el cauce principal del Alto Taibilla tiene en gran parte de su recorrido un carácter permanente, y ello le otorga una mayor capacidad de evacuación de sedimentos.

\section{Conclusiones}

El índice de conectividad desarrollado aquí indica una disminución de la conectividad de sedimentos a escala de cuenca entre los escenarios de uso de 1956 y 2006 en el Alto Taibilla. La evolución de la cubierta vegetal, hacia superficies más protegidas influye considerablemente en esta disminución de la conectividad de sedimentos de las laderas a los cauces. Tanto la construcción de diques en algunas subcuencas como las barreras geomorfológicas (barras, abanicos, nivelaciones) generadas localmente influyen también en la disminución de la conectividad sedimentológica en el período estudiado. Aunque los elementos geomorfológicos, provocan un efecto similar al de los diques de retención de sedimentos, los primeros dan lugar a respuestas sedimentarias más adaptadas en ritmos a los cambios temporales y espaciales de la cuenca de drenaje.

En este trabajo piloto la modelización del Índice de Conectividad de Cuenca $(C C I)$ propuesto para evaluar la conectividad de sedimentos a partir de la combinación de factores hidro-geomorfológicos es una metodología sencilla y de fácil aplicación. El CCI reúne principios empíricos confirmados con el trabajo de campo, es adaptable a la disponibilidad de datos y a diferentes ambientes desde una perspectiva holística. Su aplicación en cuencas de diferentes tamaños, características ambientales y en diferentes 
escenarios temporales podría servir para analizar más ampliamente su validez como herramienta de análisis en la respuesta sedimentaria de cuencas de drenaje.

\section{Agradecimientos}

Nuestro agradecimiento a la Fundación Séneca (Región de Murcia) por el apoyo recibido en el inicio del trabajo a través del proyecto ESUMA 11859/P/09. Joris de Vente agradece el contrato 'Juan de la Cierva' (JCI-2011-08941) recibido del Ministerio de Ciencia e Innovación.

\section{Referencias bibliográficas}

Ambroise, B. 2004. Variable 'active' versus 'contributing' areas or periods: a necessary distinction. Hydrological Processes 18, 1149-1155.

Beguería, S., López-Moreno, J.I., Gómez-Villar, A., Rubio, V., Lana-Renault, N., García-Ruiz, J.M. 2006. Fluvial adjustments to soil erosion and plant cover changes in the Central Spanish Pyrenees. Geografiska Annaler, Series A: Physical Geography 88, 177-186.

Boix-Fayos, C., Barberá, G.G., López-Bermúdez, F., Castillo, V.M. 2007. Effects of check dams, reforestation and land-use changes on river channel morphology: Case study of the Rogativa catchment (Murcia, Spain). Geomorphology 91, 103-123.

Boix-Fayos, C., De Vente, J., Martínez-Mena, M., Barberá, G.G., Castillo, V. 2008. The impact of land use change and check-dams on catchment sediment yield. Hydrological Processes $22,4922-4935$.

Borselli, L., Cassi, P., Torri, D. 2008. Prolegomena to sediment and flow connectivity in the landscape: A GIS and field numerical assessment. Catena 75, 268-277.

Bracken, L.J., Croke, J. 2007. The concept of hydrological connectivity and its contribution to understanding runoff-dominated geomorphic systems. Hydrological Processes 21, 1749-1763.

Brierley, G.J., Fryi, K., Jain, V. 2006. Landscape connectivity: The geographic basis of geomorphic applications. Area 38, 165-174.

Brown, C.B. 1943. Discussion of Sedimentation in reservoirs, by J. Witzig. Transactions of the American Society of Civil Engineers 69, 1493-1500.

Cammeraat, L.H. 2002. A review of two strongly contrasting geomorphological systems within the context of scale. Earth Surface Processes and Landforms 27, 1201-1222.

Cantón, Y., Solé-Benet, A., de Vente, J., Boix-Fayos, C., Calvo-Cases, A., Asencio, C., Puigdefábregas, J. 2011. A review of runoff generation and soil erosion across scales in semiarid south-eastern Spain. Journal of Arid Environments 75, 1254-1261.

Coppus, R., Imeson, A.C. 2002. Extreme events controlling erosion and sediment transport in a semi-arid sub-andean valley. Earth Surface Processes and Landforms 27, 1365-1375.

De Vente, J., Boix-Fayos, C., Blas-Larrosa, J., González-Barberá, G., Castillo, V. 2007. Restauración Hidrológico-Forestal: Efectos Sobre el Ciclo Hidrológico. Cuenca Hidrográfica del Río Segura. Informe sobre la Clasificación de Usos del Suelo, LAI y Profundidad de Raíces, CEBAS, Murcia.

De Vente, J., Poesen, J., Verstraeten, G., Van Rompaey, A., Govers, G. 2008. Spatially distributed modelling of soil erosion and sediment yield at regional scales in Spain. Global and Planetary Change 60, 393-415.

Dietrich, W.E., Belligi, D.G., Sklar, L.S., Stock, J.D., Heimsath, A.M., Roering, J.J. 2003. Geomorphic transport laws for predicting landscape form and dynamics. Prediction in 
Geomorphology. Geophysical Monograph 135. American Geophysical Union, Washington, $30 \mathrm{pp}$.

Eastman, J.R. 2003. IDRISI Kilimanjaro Guide to GIS and Image Processing, Clark Labs, Worcester.

Faulkner, H., Alexander, R., Zukowskyj, P. 2008. Slope-channel coupling between pipes, gullies and tributary channels in the Mocatán catchment badlands, Southeast Spain. Earth Surface Processes and Landforms 33, 1242-1260.

Fryirs, K., Brierley, G.J. 1999. Slope-channel decoupling in Wolumla catchment, New South Wales, Australia: the changing nature of sediment sources following European settlement. Catena 35, 41-63.

Fryirs, K., Brierley, G.J. 2001. Variability in sediment delivery and storage along river courses in Bega catchment, NSW, Australia: implications for geomorphic river recovery. Geomorphology $38,237-265$.

Fryirs, K., Brierley, G.J. 2005. Practical application of the River Styles ${ }^{\circledR}$ framework as a tool for catchment-wide river management: A case study from Bega catchment, New South Wales, $230 \mathrm{pp}$.

Fryirs, K.A., Brierley, G.J., Preston, N.J., Kasay, M. 2007a. Buffers, barriers and blankets: The (dis)connectivity of catchment-scale sediment cascades. Catena 70, 49-67.

Fryirs, K.A., Brierley, G.J., Preston, N.J., Spencer, J. 2007b. Catchment-scale (dis)connectivity in sediment flux in the upper Hunter catchment, New South Wales, Australia. Geomorphology 84, 297-316.

Gallart, F., Balasch, C., Reguiés, D., Soler, M. 2005. Catchment dynamics in a Mediterranean mountain environment: the Vallcebre research basins (South Eastern Pyrenees). II: Temporal and spatial dynamics of erosion and stream sediment transport. En Catchment Dynamics and River Processes: Mediterranean and other climate regions, C. García, R. Batalla (eds.), Elsevier, Amsterdam, pp. 17-28.

García-Ruiz, J.M., Lana-Renault, N., Beguería, S., Lasanta, T., Regüés, D., Nadal-Romero, E., Serrano-Muela, P., López-Moreno, J.I., Alvera, B., Martí-Bono, C., Alatorre, L.C. 2010. From plot to regional scales: Interactions of slope and catchment hydrological and geomorphic processes in the Spanish Pyrenees. Geomorphology 120, 248-257.

Hancock, G.R., Coulthard, T.J. 2012. Channel movement and erosion response to rainfall variability in southeast Australia. Hydrological Processes 26, 663-673.

Hooke, J. 2003. Coarse sediment connectivity in river channel systems: a conceptual framework and methodology. Geomorphology 56, 79-94.

Hooke, J. 2006. Human impacts on fluvial systems in the Mediterranean region. Geomorphology 79, 311-335.

IGME 1980. Hojas de la Serie MAGNA 888 (Yetas), 899 (Yetas de Abajo), 909 (Nerpio) y 910 (Caravaca). Instituto Geológico y Minero de España, Madrid, 34 pp., 54 pp., 36 pp, 34 pp.

Kasai, M., Brierley, G.J., Page, M.J., Marutani, T., Trustrum, N.A. 2005. Impacts of land use change on patterns of sediment flux in Weraamaia catchment, New Zealand. Catena 64, $27-60$.

Keesstra, S.D., Van Dam, O., Verstraeten, G., Van Huissteden, J. 2009. Changing sediment dynamics due to natural reforestation in the Dragonja catchment, SW Slovenia. Catena 78, 60-71.

Kirkby, M.J., Bracken, L., Reany, S. 2002. The influence of land use, soils and topography on the delivery of hillslope runoff to channels in SE Spain. Earth Surface Processes and Landforms 27, 1459-1473.

Lana-Renault, N. 2011. El efecto de los cambios de cubierta vegetal en la respuesta hidrológica y sedimentológica de áreas de montaña: la cuenca experimental de Arnás, Pirineo central. Consejo de Protección de la Naturaleza de Aragón, Zaragoza, 189 pp. 
Lane S.N., Brookes, C.J., Kirkby, M.J., Holden, J. 2004. A network-index-based version of TOPMODEL for use with high-resolution digital topographic data. Hydrological Processes 18 , 191-201.

Lesschen, J.P., Kok, K., Verburg, P.H., Cammeraat, L.H. 2007. Identification of vulnerable areas for gully erosion under different scenarios of land abandonment in Southeast Spain. Catena 71, 110-121.

López-Vicente, M., Poesen, J., Navas, A., Gaspar, L. 2013. Predicting runoff and sediment connectivity and soil erosion by water for different land use scenarios in the Spanish Pre-Pyrenees. Catena 102, 62-67.

McHug, M., Wood, G., Walling, D.E., Morgan, R., Zhang, Z., Anthony, S., Hutchins, M. 2002. Prediction of sediment delivery to watercourses from land, Phase II. R\&D Technical Report no. P2-209, National Soil Resource Centre, Cranfield University, Cranfield.

Meerkerk, A.L., Van Wesemael, B., Bellin, N. 2009. Application of connectivity theory to model the impact of terrace failure on runoff in semi-arid catchments. Hydrological Processes 23, 2792-2803.

Michaeliodes, K., Wainwright, J. 2002. Modelling the effects of hillslope-channel coupling on catchment hydrological response. Earth Surface Processes and Landforms 27, 1441-1457.

Prosser, I.P., Rustomji, P. 2000. Sediment transport capacity relations for overland flow. Progress in Physical Geography 24, 179-193.

Quiñonero-Rubio, J.M., Boix-Fayos, C., Nadeu, E., de Vente, J. En revisión. Evaluation of the effectiveness of land use change and hydrological forest restoration works to reduce catchment sediment yield. Land Degradation and Development.

Reaney, S.M. 2008. The use of agent based modelling techniques in hydrology: determining the spatial and temporal origin of channel flow in semi-arid catchments. Earth Surface Processes and Landforms 33, 317-327.

Reaney, S.M., Bracken, L.J., Kirkby, M.J. 2007. Use of the Connectivity of Runoff Model (CRUM) to investigate the influence of storm characteristics on runoff generation and connectivity in semi-arid areas. Hydrological Processes 21, 894-906.

Sandercock, P.J., Hooke, J.M. 2011. Vegetation effects on sediment connectivity and processes in an ephemeral channel in SE Spain. Journal of Arid Environments 75, 239-254.

Sougnez, N., Van Wesemael, B., Vanacker, V. 2011. Low erosion rates measured for steep, sparsely vegetated catchments in southeast Spain. Catena 84, 1-11.

Van Oost, K., Govers, G., Desmet, P.J.J. 2000. Evaluating the effects of changes in landscape structure on soil erosion by water and tillage. Landscape Ecology 15, 577-589.

Van Rompaey, A. J.J., Verstraeten, G., Van Oost, K., Govers, G., Poesen, J. 2001. Modelling mean annual sediment yield using a distributed approach. Earth Surface Processes and Landforms 26, 1221-1236.

Verstraeten, G., Van Oost, K., Van Rompaey, A., Poesen, J., Govers, G. 2002. Evaluating an integrated approach to catchment management to reduce soil loss and sediment pollution through modelling. Soil Use and Management 19, 386-394.

Verstraeten, G., Prosser, I.P., Fogarty, P. 2007. Predicting the spatial patterns of hillslope sediment delivery to river channels in the Murrumbidgee catchment, Australia. Journal of Hydrology 334, 440-454.

Vigiak, O., Borselli, L., Newham, L.T.H., McInnes, J., Roberts, A.M. 2012. Comparison of conceptual landscape metrics to define hillslope-scale sediment delivery ratio. Geomorphology $138,74-88$.

Walling, D.E. 1983. The sediment delivery problem. Journal of Hydrology 65, 209-237. 